\title{
Modelos de Distribuição de Espécies em Estratégias para a Conservação da Biodiversidade e para Adaptação Baseada em Ecossistemas Frente a Mudanças Climáticas
}

\author{
Species Distribution Models (SDM) in Biodiversity Conservation Strategies and \\ Climate Change Ecosystem Based Adaptation
}

\author{
Adriano Pereira Paglia ${ }^{1 *}$, Daniella Teixeira de Rezende1, Ingrid Koch², \\ Alessandra Rocha Kortz ${ }^{2} \&$ Camila Donatti ${ }^{3}$
}

\begin{abstract}
${ }^{1}$ Laboratório de Ecologia e Conservação, Departamento de Biologia Geral, Instituto de Ciências Biológicas - ICB, Universidade Federal de Minas Gerais - UFMG, Belo Horizonte, MG, Brasil

${ }^{2}$ Departamento de Ciências Biológicas, Centro de Ciências e Tecnologias para a Sustentabilidade - CCTS, Universidade Federal de São Carlos - UFSCar, Sorocaba, SP, Brasil

${ }^{3}$ Conservation International, Arlington, VA, USA
\end{abstract}

\section{Modelagem de Distribuição de Espécies em Ecologia e Biologia da Conservação}

O conhecimento da distribuição geográfica é fundamental para embasar estudos evolutivos e ecológicos das espécies. Porém, a sua delimitação é um desafio devido à existência de concentração espacial desigual de informação de distribuição das espécies, alto custo de trabalhos de campo, e às variações que ocorrem nessa distribuição ao longo do tempo. Tendo em vista essa problemática, os métodos de Modelagem de Distribuição de Espécies (MDE) surgiram como uma grande contribuição para os estudos realizados nessas áreas. Os MDEs são muito utilizados, por exemplo, para priorizar áreas para conservação (Nóbrega \& De Marco Junior 2011), discutir padrões biogeográficos (Werneck et al. 2012) e, com a disponibilização de dados modelados de clima passado e futuro, prever mudanças na distribuição dos organismos ao longo do tempo (Bonnacorso et al. 2006).

A teoria do nicho ecológico é o principal fundamento para o desenvolvimento de MDEs (Peterson 2011) considerando que indivíduos de uma mesma espécie respondem a um conjunto de recursos e condições que definem sua distribuição (Grinnell 1917; Hutchinson 1957). Os MDEs são baseados, principalmente, nas condições ambientais e são gerados a partir de um conjunto de regras que vão

\footnotetext{
*Autor para correspondência: Adriano Pereira Paglia Departamento de Biologia Geral, Instituto de Ciências Biológicas, Universidade Federal de Minas Gerais - UFMG, Av. Antônio Carlos, 6627, Pampulha, CEP 31270-901, Belo Horizonte, MG, Brasil

E-mail: apaglia@icb.ufmg.br
}

desde soluções matemáticas mais simples (Distância Euclidiana, BIOCLIM), passando por ajustes estatísticos (Modelos Lineares Generalizados - GLM, Modelos Aditivos Generalizados - GAM) até algoritmos derivados de inteligência artificial e busca (Maxent, GARP, Redes Neurais). Basicamente, o que estes algoritmos calculam é a similaridade ambiental entre os locais de ocorrência conhecidos para a espécie e outras regiões ainda desconhecidas. Ao final, os locais de maior similaridade são considerados como áreas de alta probabilidade de ocorrência.

A União Internacional para Conservação da Natureza, IUCN, utiliza um conjunto de critérios objetivos e quantitativos para categorizar o status de conservação de espécies em escala global (IUCN 2001). Um desses critérios (critério B) diz respeito ao tamanho atual da distribuição de uma espécie e como esse espaço geográfico tem sido alterado por ação antrópica. Assim, a categorização da situação de ameaça depende de quão bem estimada é a distribuição geográfica das espécies em avaliação. Os métodos estabelecidos pela IUCN para estimar a distribuição de espécie são o tamanho da Extensão de Ocorrência (EdO) ou da Área de Ocupação (AdO). A EdO é o mínimo polígono convexo que engloba as localidades conhecidas de uma espécie e geralmente produz superestimativas da distribuição geográfica ao incorporar áreas que não são adequadas para a espécie. A AdO é a soma do tamanho das células ocupadas pela espécie em um grid espacial. A AdO tenta corrigir erros de superestimativa da EdO, porém pode levar a erros de omissão, desconsiderando 
áreas potenciais, mas não amostradas, de ocorrência da espécie e subestimando o tamanho da distribuição. Além das armadilhas de superestimar ou subestimar o tamanho da distribuição geográfica os métodos tradicionais utilizados pela IUCN são sensíveis ao incremento da informação biológica advinda da geração de novos dados de ocorrência pontual, que por sua vez são uma das principais causas de downgrade na categoria de ameaça de uma espécie (Paglia \& Fonseca 2009).

Considerando a distribuição enviesada dos dados de ocorrência ou mesmo a pequena quantidade de informação de distribuição de muitas espécies ameaçadas, a MDE pode contribuir diminuindo erros de categorização do status de ameaça dessas espécies. Indo além do documento base de categorias e critérios (IUCN 2001), a IUCN recentemente avaliou a utilização de mapas de distribuição gerados por modelagem preditiva como ferramenta adicional para estimativas do tamanho da AdO (IUCN 2010). Por exemplo, Jimenez-Alfaro et al. (2012) utilizaram MDE para uma espécie de planta herbácea europeia ameaçada de extinção e produziram estimativas da AdO potencial menores, mais robustas e menos afetada pelo método de amostragem em comparação com as estimativas pelo método tradicional.

Uma das características desejadas dos MDEs é a transferabilidade, ou seja, a possibilidade de indicar áreas potenciais de ocorrência das espécies além dos locais já conhecidos. Para espécies ameaçadas de extinção, é importante gerar, de maneira rápida e eficiente, novas informações sobre suas distribuições geográficas e sobre o status de suas populações. Modelos de Distribuição Potencial podem ser úteis como ferramenta direcionadora de esforços amostrais para espécies ameaçadas. Ainda são poucos os trabalhos envolvendo coletas em novas áreas indicadas por MDEs e validação dos modelos em campo, porém os resultados destes estudos se mostraram satisfatórios (Siqueira et al. 2009).

A redução do tamanho da área de distribuição geográfica é um dos fatores que levam espécies à extinção, e o principal vetor de perda de habitat no Brasil é a supressão da vegetação nativa. A estratégia mais utilizada para reduzir a perda de habitat é a criação de Unidades de Conservação de Proteção Integral. O Planejamento Sistemático para Conservação (PSC) tem sido utilizado mais recentemente para indicar as melhores áreas para criação de novas UCs e modelagem preditiva é parte do processo ao estimar a distribuição potencial de espécies-alvo para conservação. Porém, com cenários projetados de mudanças climáticas futuras tem havido um questionamento sobre a manutenção da eficiência das UCs atuais em proteger espécies ameaçadas em regiões mais afetadas por alterações climáticas (Wiens et al. 2011). Qual seria, portanto o nível de vulnerabilidade de espécies e ecossistemas a cenários futuros de mudanças climáticas? MDEs podem ser úteis para essa análise de vulnerabilidade? Seria possível definir ações de adaptação a novas condições climáticas baseadas nos resultados dos MDEs?

\section{MDEs, Vulnerabilidade a Mudanças Climáticas e Adaptação Baseada nos Ecossistemas}

Adaptação às mudanças climáticas é definida pelo Painel Intergovernamental sobre mudanças climáticas (IPCC) como

[...] ajustamentos nos sistemas naturais ou humanos, em resposta a estímulos climáticos presentes ou esperados, ou os seus efeitos, que moderam os danos ou tiram partido das oportunidades úteis [...] (IPCC 2007, p.869).

Uma das estratégias usadas na adaptação às mudanças climáticas é a chamada Adaptação baseada nos Ecossistemas $(\mathrm{AbE})$. A AbE refere-se às estratégias de adaptação que integram o uso da biodiversidade e os serviços ecossistêmicos como uma forma de ajudar as pessoas a se adaptarem aos impactos adversos das mudanças climáticas. AbE é vista como um grande contribuidor do bem estar humano e é uma ligação óbvia entre a necessidade de ecossistemas saudáveis e a necessidade do aumento da capacidade adaptativa das pessoas que dependem desses ambientes. Essa abordagem busca alinhar diferentes objetivos, como desenvolvimento econômico e conservação da biodiversidade.

O primeiro passo para a identificação das ações de AbE é a execução de uma análise de vulnerabilidade às mudanças climáticas em uma dada região, através da qual são identificados os principais impactos das mudanças climáticas sobre populações humanas e sobre a biodiversidade. Muitas das análises dos impactos das mudanças climáticas para a conservação da biodiversidade são focadas na distribuição das espécies, uma vez que o clima constitui parte importante do conjunto de condições abióticas que delimitam a área de ocorrência de uma determinada espécie. Pelo fato dos ecossistemas serem uma peça chave da $\mathrm{AbE}$, a MDE é frequentemente usada como parte dessa análise de vulnerabilidade. As espécies usadas na análise podem ser aquelas utilizadas pelas comunidades locais como fonte de renda, espécies comercialmente importantes, espécies invasoras, espécies ameaçadas, espécies-chave para processos ecológicos fundamentais e espécies típicas de fitofisionomias em particular que, juntas, podem nos dar informações sobre um dado processo (i.e. ressurgência e estoques pesqueiros) e sobre a resiliência de um dado ecossistema frente às mudanças climáticas.

Uma das maneiras de se avaliar as consequências das mudanças climáticas para a distribuição de espécies é utilizar MDE para visualizar as alterações na disponibilidade de áreas com condições ambientais adequadas para a ocorrência de determinada espécie ou grupo de espécies, frente às alterações no clima. As projeções dos modelos de distribuição potencial para cenários climáticos distintos baseiam-se no conceito de conservantismo de nicho (Peterson 2011) e a projeção no ambiente geográfico do conjunto de condições climáticas exigidas atualmente pelas espécies para cenários futuros permite simular, desta forma, 
quais serão as áreas adequadas para a ocupação por estas espécies nestes cenários. Para que estes modelos possam ser gerados é necessário reunir os dados de ocorrência atual das espécies e as camadas ambientais com informações contínuas de clima interpoladas para cenários futuros. Atualmente existem vários métodos que geram camadas climáticas com dados contínuos para cenários climáticos do futuro e estes podem considerar situações climáticas alternativas, como maior ou menor aumento na temperatura e variações na precipitação (ver Franklin et al. 2012 para uma revisão).

Dessa maneira, MDE considerando a distribuição potencial em cenários futuros, tem se mostrado útil no planejamento de ações de conservação, a partir da indicação de áreas prioritárias (Nóbrega \& De Marco Junior 2011), chamando a atenção para espécies ou ecossistemas ameaçados (Marini et al. 2009).

\section{Estudo de Caso: "Projeto IKI" e Como a Distribuição de Espécies Pode Nos Ajudar em Esforços de Adaptação}

O projeto IKI é financiado pela Iniciativa Internacional do Clima (IKI) do Ministério do Meio Ambiente, da Proteção da Natureza e da Segurança Nuclear da Alemanha (BMU). Esse projeto visa a implementação de ações de Adaptação baseada nos Ecossistemas (AbE) como forma de melhorar as condições de vida e conservar a biodiversidade frente à mudanças do clima em regiões marinhas, terrestres e costeiras em três áreas geográficas distintas: Brasil, África do Sul e Filipinas. No Brasil, o projeto está sendo executado sob a coordenação da "Conservation International" e desenvolvido na interface dos ambientes marinho e terrestre, no Corredor Central da Mata Atlântica, com foco mais específico para a região de Abrolhos, no sul da Bahia. Essa área abriga os maiores remanescentes da Mata Atlântica na região nordeste, os maiores e mais ricos recifes de corais do Atlântico Sul. Cerca de 500.000 pessoas pessoas dependem dos serviços ecossistêmicos nessa região, entretanto a sobrepesca, os desmatamentos para a criação de gado, agricultura e a ocupação humana estão impactandoos ecossistemas e os tornando vulneráveis às mudanças climáticas.

Nesse contexto, a Conservation International e parceiros estão conduzindo uma análise de vulnerabilidade às mudanças climáticas, a qual será seguida pela implementação de estratégias de AbE nos sistemas terrestres e marinhos dessa região.Como parte da análise de vulnerabilidade, 50 espécies de plantas e 50 espécies de vertebrados foram escolhidas para terem suas distribuições modeladas em condições presentes e futuras. As espécies de plantas usadas na modelagem foram aquelas típicas de determinadas fitofisionomias como a floresta ombrófila densa, restinga, e mangue, e suas distribuições futuras nos fornecem uma base para entendermos se tais fisionomias irão expandir ou contrair, permitindo assim uma base para um planejamento que leve em conta as mudanças climáticas. Outras espécies usadas na modelagem foram aquelas economicamente importantes na região (como a piaçava, Attalea funifera), as quais nos fornecem informações que nos ajudam a identificar quais comunidades tradicionais estarão mais vulneráveis às mudanças climáticas.

Em relação às espécies de vertebrados, o foco é sobre aquelas que atuam como importantes dispersores de sementes e que são importantes para a manutenção das diferentes fitofisionomias estudadas e dos processos estruturadores da floresta. As distribuições futuras dessas espécies são também cruciais para nos guiar nas ações de adaptação, como por exemplo, na avaliação dos custos da restauração florestal em áreas com diminuição prevista de espécies dispersoras de sementes ou na identificação de áreas que podem ser usadas como corredores ecológicos.

Além de avaliar como a perda de espécies de árvores e dos vertebrados dispersores de suas sementes pode afetar a extensão da cobertura das diferentes fitofisionomias da Mata Atlântica no Corredor Central, a modelagem de distribuição potencial para essas 100 espécies pode fornecer subsídios para a avaliação do status de conservação ao estimar a diminuição prevista da extensão de ocorrência e também analisar quão eficiente é o atual sistema de Unidades de Conservação de Proteção Integral na região sul da Bahia e no Espírito Santo. Nossas análises preliminares indicam que parcela significativa das espécies modeladas, inclusive daquelas que já são consideradas ameaçadas de extinção, terão suas áreas de distribuição geográfica afetadas em vários dos cenários futuros que foram empregados na análise.

Os MDEs podem fornecer importante contribuição para a elaboração e implantação de estratégias de conservação in situ realizadas tanto por organizações conservacionistas locais quanto por organismos internacionais atuando em escalas mais amplas, conferindo um caráter mais aplicado a uma ferramenta analítica que surgiu para tentar responder questões básicas acerca de padrões biogeográficos e ecológicos. De qualquer maneira, em exercícios de MDE o cuidado com a seleção das espécies a serem modeladas, a definição clara dos objetivos da modelagem, o rigor metodológico na compilação e avaliação da qualidade dos dados pontuais de ocorrência das espécies, a seleção dos melhores modelos preditivos e análise das incertezas associadas a eles e a eventual validação dos resultados das modelagens, são fundamentais para a qualidade do produto gerado, seja ele de caráter aplicado ou não.

\section{Agradecimentos}

Os autores agradecem a Paulo De Marco Jr pelo convite e pelas contribuições ao manuscrito original. A Lucio Bedê pelas discussões sobre adaptação baseada em ecossistemas. A.P. Paglia agradece ao CNPq (processo 480283/2011-7) e à $\mathrm{PRPq} / \mathrm{UFMG}$ pelo apoio aos projetos de pesquisa e à FAPEMIG pelo auxílio do Programa Pesquisador Mineiro (processo CRA - PPM-00109-12).C. Donatti agradece o Ministério do Meio Ambiente, da Proteção da Natureza e 
da Segurança Nuclear da Alemanha (BMU) pelo apoio ao projeto IKI. D.T. Rezende é bolsista da CAPES.

\section{Referências}

Bonnacorso E, Koch I \& Peterson T, 2006. Pleistocene fragmentation of Amazon species'ranges. Diversity and Distributions, 12(2):157-164. http://dx.doi. org/10.1111/j.1366-9516.2005.00212.x

Franklin J et al., 2012. Modeling plant species distributions under future climates: how fine scale do climate projections need to be? Global Change Biology. Early View. http://dx.doi. org/10.1111/gcb.12051

Grinnell J, 1917. Field tests of theories concerning distributional control. The American Naturalist, 51(602):115-128. http:// dx.doi.org/10.1086/279591

Hutchinson GE, 1957. Concluding Remarks. Cold Spring Harbour Symposium on Quantitative Biology, 22:415-427.

Intergovernmental Panel on Climate Change - IPCC, 2007. Climate Change 2007: Impacts, Adaptation and Vulnerability. Contribution of Working Group II to the Fourth Assessment Report of the Intergovernmental Panel on Climate Change. Cambridge: Cambridge University Press. 976 p.

International Union for Conservation of Nature - IUCN, 2001. IUCN Red List Categories and Criteria. Version 3.1. Gland; Switzerland; Cambridge: IUCN. IUCN Species Survival Commission.

International Union for Conservation of Nature - IUCN. Standards and Petitions Subcommittee, 2010. Guidelines for Using the IUCN Red List Categories and Criteria. Version 8.1. Standards and Petitions Subcommittee. Available from: $<$ http://intranet.iucn.org/webfiles/doc/SSC/RedList/ RedListGuidelines.pdf>.
Jimenez-Alfaro B, Draper D \& Nogués-Bravo D, 2012. Modeling the potential area of occupancy at fine resolution may reduce uncertainty in species range estimates. Biological Conservation, 147(1):190-196. http://dx.doi.org/10.1016/j. biocon.2011.12.030

Marini MA et al., 2009. Predicted climate-driven bird distribution changes and forecasted conservation conflicts in a Neotropical Savanna. Conservation Biology, 142(12):3039-3050. http:// dx.doi.org/10.1016/j.biocon.2009.08.002

Paglia AP \& Fonseca GAB, 2009. Assessing changes in the conservation status of threatened Brazilian vertebrates. Biodiversity and Conservation, 18(13):3563-3577. http:// dx.doi.org/10.1007/s10531-009-9660-0

Nóbrega CC \& De Marco Junior P, 2011. Unprotecting the rare species: a niche-based gap analysis for odonates in a core Cerrado area. Diversity and Distributions, 17(3):491-505. http://dx.doi.org/10.1111/j.1472-4642.2011.00749.x

Peterson AT, 2011. Ecological niche conservatism: a time-structured review of evidence. Journal of Biogeography, 38(5):817-827. http://dx.doi. org/10.1111/j.1365-2699.2010.02456.x

Siqueira MF et al., 2009. Something from nothing: Using landscape similarity and ecological modeling to find rare plant species. Journal for Nature Conservation, 17(1):25-32. http://dx.doi.org/10.1016/j.jnc.2008.11.001

Werneck FP et al., 2012. Deep diversification and long-term persistence in the South American dry 'dry diagonal': integrating continent-wide phylogeography and distribution modeling of geckos. Evolution, 66(10):3014-3034. PMid:23025595. http://dx.doi. org/10.1111/j.1558-5646.2012.01682.x

Wiens JA, Nathaniel ES \& Jongsomjit D, 2011. Protected areas in climate space: What will the future bring? Biological Conservation, 144(8):2119-2125. http://dx.doi.org/10.1016/j. biocon.2011.05.002 